\section{Counseling Prior Contraception: Is It a Provider Failure or Patient Failure?}

\section{Abstract}

Unintended pregnancy is not only a major health problem, but are also a great social and financial burden on societies and countries. There are about 75 million unwanted pregnancies per year according to WHO statistics. When abortions were included, unintended pregnancies increased to 2.65 million, or $49 \%$ of all pregnancies. Counseling prior contraception is a mandatory or a must to prevent these unintended pregnancies and their related risks. Unfortunately counseling is a deficient process in third world countries and usually made by young medical staffs or sometimes by paramedical personnel or not present at all. In this perspective, issues for deficient counseling and how patients select the method of contraception are addressed.

Keywords: Pregnancy, WHO, Contraception, Maternal

Received: January 30, 2017, Accepted: February 08, 2017 Published: February 15, 2017

\section{Introduction}

Unintended pregnancies had many health related problems affecting women when no proper counseling was done either at the time of delivery or later at time of contraceptive method selection [1].

Unintended pregnancy threaten woman's health as patient may have existing health problems aggravated or poorly controlled during pregnancy. Moreover, lack of support and financial resources affect proper antenatal care to achieve healthy pregnancy and raise a healthy child. Another serious issue to be addressed which is the unsafe abortion, as most of these patients opt to abortion to terminate the nightmare she and her family feel every day. Unsafe abortions had many morbidities and maternal mortality was found to be very high due to these unsafe procedures [2].

Appropriate prevention is the magic cure in all aspects. Prevention comes and starts by appropriate counseling at contraceptive clinics. Unfortunately, this issue is neglected or underestimated in third world countries posing more economic, social and health burdens [3].

The wide range of contraceptive methods, advantages and disadvantages, suitability and effectiveness of each method for each patient is a major task for the health care providers. It is important to consider patient choices and human rights, not obligations and oppression to use one method only. It is well known that when patient share in selection of a method, she is motivated to use it consistently and correctly. It is the counseling,

\section{Ayman Shehata Dawood MD}

Department of Obstetrics and Gynecology, Faculty of Medicine, Tanta University, Egypt
Corresponding author:
Ayman Shehata Dawood

ayman.dawood@med.tanta.edu.eg.

Department of Obstetrics and Gynecology, Faculty of Medicine, Tanta University, Egypt.

Tel: +201020972067

Citation: Dawood MD AS. Counseling Prior Contraception: Is It a Provider Failure or Patient Failure?. J Contracept Stud. 2017, 2:1 where the health care provider discusses all available methods, price, application, side effects, efficacy, and the best method for each patient according to age, medical condition and pregnancy desire [4].

An important issue to be put in mind that some religious and social areas had negative images and concepts regarding family planning and contraception. These issues need to be addressed and solved by both community religious leaders and health care provider. These are not negligible issues, as they affect compliance and continuation of birth control methods in many areas of the world [5].

Another ethical issue is that promotion of health care provider to one method for other methods. This is an important issue in third world countries, due to economic and financial constraints [6].

Patients mostly know about contraceptive methods from previous experience and compliance with previously tired methods, her relatives, her neighbors, and from social media. The husbands more or less share in counseling by relation of method selected to sexual behavior and men's health checks as well. This will enforce users' compliance with birth control methods [7].

Unfortunately, counseling is underestimated in low socioeconomic countries and provided by low experience personnel or even non experienced ones provide such important issue [8].

All the previously mentioned issues make the counseling a real challenge in front of health care providers and patients equally. 
Success of counseling to match each for certain allocated method is considered provider success. This success never be complete unless the patient was motivated and use the method in the best way correctly and concisely [9].

Patient's culture, education and social state are common determinants for patient's success or failure and are the key to compliance and efficacy. Understanding the needs and characteristics of the individual patient can help the health-care provider to direct her towards the method that will best suit her needs in terms of efficacy, safety and ease of use [10].

\section{Conclusion}

Counseling prior contraception must be given more attention in low socio-economic countries. Counseling is a great challenge to both health care provider and patient as well. Sharing woman in decision of a specific method potentiate and motivate women for more compliance and success of such method.

\section{Conflicts of interest}

No conflicts of interest are present. 


\section{References}

1 Shah IH, Chandra MV (2007) Inequalities and unwanted fertility in developing countries. Bull World Health Organ. Vol-85, pp: 86.

2 Zapata LB, Murtaza S, Whiteman MK, Jamieson DJ, Robbins CL, et al. (2015) Contraceptive counseling and postpartum contraceptive use. Journal of Obstetrics and Gynecology, 212: 1-8.

3 Merckx M, Donders GG, Grandjean P, Van de Sande T, Weyers S, et al. (2011) Does structured counselling influence combined hormonal contraceptive choice? European Journal of Contraception and Report Health Care, 16: 418-429.

4 Bitzer J, Cupanik V, Fait T, Gemzell D, Grob P (2013) Factors influencing women's selection of combined hormonal contraceptive methods after counseling in 11 countries: results from a sub analysis of the CHOICE study. European Journal of Contraceptive Report Health Care, 18: 372-380.

5 Merki-Feld GS, Gruber IM (2014) Broad counseling for adolescents about combined hormonal contraceptive methods: the choice study. J Adolesc Health 54: 404-9.
6 Lopez LM, Grey TW, Tolley EE, Chen M. Brief educational strategies for improving contraception use in young people. Cochrane Database Syst Rev.

7 Kofinas JD, Varrey A, Sapra KJ, Kanj RV, Chervenak FA, et al.(2014) Adjunctive social media for more effective contraceptive counseling: a randomized controlled trial. Obstet Gynecol.: 763-770.

8 Levy K, Minnis AM, Lahiff M, Schmittdiel J, Dehlendorf C, et al. (2015) Bringing patients' social context into the examination room: an investigation of the discussion of social influence during contraceptive counseling. Women's Health Issues, 25: 13-21.

9 Modesto W, Bahamondes MV, Bahamondes L (2014) A randomized clinical trial of the effect of intensive versus non-intensive counseling on discontinuation rates due to bleeding disturbances of three longacting reversible contraceptives. Human Report, 29: 1393-1399.

10 Mc Nicholas C, Madden T, Secura G, Peipert JF (2014) The contraceptive choice project round up: what we did and what we learned. Clinical Obstetrics Gynecology, 57: 635-643. 